\title{
COMPARISON OF VARIOUS EEG ELECTRODE PLACEMENT SYSTEMS TO DETECT EPILEPTIFORM ABNORMALITIES IN INFANTS
}

Sajeesh Parameswaran ${ }^{1}$, Thankappakurup Vijayamma Anil Kumar ${ }^{1}$, Ajith Mohan ${ }^{1}$, John Thomas ${ }^{1}$, Nikhil Sajeev ${ }^{1}$, Kamala Swarnam $^{2}$, Ananthanarayana Marthanda Pillai ${ }^{3}$

Correspondence: psajeesh@gmail.com

${ }^{1}$ Department of Neurology, Ananthapuri Hospitals and Research Institute,Trivandrum,India

${ }^{2}$ Department of Pediatrics, Ananthapuri Hospitals and Research Institute,Trivandrum,India

${ }^{3}$ Department of Neurosurgery, Ananthapuri Hospitals and Research Institute,Trivandrum,India

\section{Article History:}

Received: Mei 4, 2020

Accepted: September 29, 2020

Published: January 1, 2021

\section{Cite this as:}

Parameswaran S, Kumar TVA, Mohan A, Thomas J, Sajeev N, Swarnam K, Pillai AM.

Comparison of various eeg

electrode placement systems to detect epileptiform abnormalities in infants. Malang Neurology Journal; 2021.7:30-33.

http://dx.doi.org/10.21776/ub.mnj .2021.007.01.7

\section{ABSTRACT}

Background: Technical difficulties in placement of whole 10-20 electrode system is not uncommon in neonates and infants. Apart from the full channel many centers uses the modified and amplitude integrated EEG montages to identify seizures.

Objective: Efficacy of standard, modified and amplitude integrated EEG electrode placement in infants to detect epileptiform abnormalities.

Methods: All routine EEGs from June 2015 to April 2018 were taken. Age $\leq 2$ years at the time of recoding was the inclusion criteria. Digital EEG was performed according to standard 10-20 electrode placement system in all patients. Abnormal EEGs were reanalyzed in different montages like A) 21 electrodes- full channel, B) 10 electrodes- modified long distance and C) 4 electrodes- (centro/parietal) respectively. Inter ictal epileptiform discharges (IEDs), seizures, periodic complexes and non-specific dysfunctions were tabulated in all patients. Full channel montages are considered bench mark for the analysis

Results: A total 129 EEGs analyzed. A) Full channel standard 21 electrodes montages could identify IEDs in 35, non-specific electrophysiological dysfunction in 9 and burst-suppression pattern in 2 EEGs. B) Modified electrode placement consists of 10 electrodes could identify IEDs only in 26 EEGs and non-specific electro physiological dysfunction in 6 EEGs. A total of 28 nonconvulsive seizures (NCS) recorded in 6 patients; 20 numbers of NCS (71.4\%) seen in modified electrode placement (B) and only 16 (57.1\%) seen in centro/parietal electrode placement (C)..

Conclusion: Standard EEG electrode placement has higher yield in detecting epileptiform abnormalities.

Keywords: EEG, electrode placement, epilepsy, infants

\section{Introduction}

EEG reading and identifying epileptiform abnormalities are very crucial in neuro-critical care managements. Limited resources, reduced EEG channels and inter observer variability are some of the factors that affects in peadiatric EEG recording. ${ }^{1}$ EEG monitoring helps to modify clinical management in majority of the patients. ${ }^{2}$ Continuous EEG or amplitude integrated EEG monitoring in high risk newborn babies certainly helps to reduce neurological morbidity and mortality by optimizing neuro care. . $^{3,4}$

Usually EEG electrodes are placed according to internationally accepted 10-20 electrode measurement system in adult and pediatric age groups. Technical difficulties in placement of whole 10-20 electrodes are not uncommon in neonates and infants; especially is intensive care units due to lot of external interferences. But treatment of electrographic seizures with the help of EEG monitoring results significant reduction in seizure morbidity. ${ }^{5}$ Placing all standard EEG electrodes in infants is very tedious task because of small head circumference and frequent movements. These practical considerations have led to make use of a two-electrode (single channel biparietal, P3$\mathrm{P} 4)$ or a four-electrode (two channel centro/fronto-parietal) derivations. ${ }^{6}$ Such approach has been questioned by clinical neurophysiologists because of their custom of recording newborns with eight to ten electrodes or even mote. ${ }^{7,8}$ However, a limited amount of evidence for, or against, the idea that decreasing the number of electrodes from a full montage would compromise performance in the clinical settings. ${ }^{9,10}$ The detection of seizures is the most generally used application of neonatal EEG monitoring. ${ }^{11}$ Apart form the full channel many centers use the modified and amplitude integrated EEG montages. We through our present analysis are trying to study the reliability of limited channel montages.

The objective of this research is to assess the efficacy of standard, modified and amplitude integrated EEG electrode placement in infants to detect epileptiform abnormalities. 


\section{Methods}

The EEG signals were collected using digital EEG amplifier (sampling frequency of $400 \mathrm{~Hz}$, Natus, Canada) with 21 electrodes positioned as per the international 10-20 standard (further details of the neonatal EEG recording method, see Vanhatalo et al. ${ }^{12}$ Apart from the 10-20 electrode placement; additional ECG, EMG and noncephalic reference electrodes were placed. All routine EEGs from June 2015 to April 2018 were included. Age < 2 years at the time recording was the inclusion criteria. We retrospectively analyzed the whole data using different montages by computer reconstruction. Our team members jointly read the EEG using different montages and marked the epileptiform abnormalities. We compared the results of the already recorded 10-20 electrode system from all the patients with the 10-electrode long distance EEG montage and a 4- electrode EEG montage s; latter were reconstructed from each recordings. ${ }^{13}$

We categorized the recordings as A) Full channel recording, B) Modified-10 electrodes and C) Limited-4 electrode montages. In (B); montages were created using Fp1/2, C3/4, O1/2 and Fz and Pz. In (C), montages were created using $\mathrm{C} 3 / 4$ and $\mathrm{P} 3 / 4$. Seizures were identified using the well-established definition; ie a distinct, abnormal electrographic event with a clear beginning and end comprising sustained, repetitively evolving spike/sharp waves or rhythmic waveforms. ${ }^{14}$ Duration and spatial evolution of all seizures were analyzed. We used the settings of paper speed of $30 \mathrm{~mm} / \mathrm{s}$, a sensitivity of 7 $\mathrm{V} / \mathrm{mm}$ with frequency cutoffs of $0.5 \mathrm{~Hz}$ (low) and $70 \mathrm{~Hz}$ (high). Apart from non-convulsive seizure (NCS); all nonspecific abnormalities and inter ictal epileptiform discharges (IEDs) were also tabulated.

We analyzed the epileptiform abnormalities in the internationally accepted 10-20 electrode placement system (A) with the modified (B) and limited (C). IEDs and NCS were tabulated in each group. Categorical data summarized using proportions and observed differences among proportions in the epileptiform abnormalities where tested using Chi square. $\mathrm{P}<0.05$ was considered significant.

\section{Results}

A total 129 EEGs analyzed. Epileptiform and nonepileptiform abnormalities were analyzed and tabulated in each groups (Table 1). Full channel (A) showed IEDs in 35 and NCS in 28 EEGs. Many of the short lasting NCS with less spatial evolution better expressed and identified only in Full electrode system A (figure 1). Modified electrode placement (B) could identify IEDs in 26 and NCS in 20. Some of the seizures can easily seen in both A and B (figure 2). The limited group (C) could identify IEDs only in 10 and NCS in 16 EEGs. Some of the ictal pattern; especially diffuse electrical onset could be seen in all montages (figure 3). This shows that epileptiform abnormalities were better seen and identified in full channel A group compare to other B and C ( Fisher exact test; significant at $\mathrm{p}<.05$ )

Similarly non-specific abnormalities also tabulated. Many of the focal slow waves were missed in the other two montages; B and C. Burst suppression pattern were easily identified in all three groups (figure 4). We also done quantitative EEG analysis using amplitude integrated EEG.

Table 1. Epileptiform and non-epileptiform abnormalities

\begin{tabular}{lcc}
\hline & Modified (B) & Limited (C) \\
\hline IEDs & $26(74.3 \%)$ & $10(28.6 \%)$ \\
NCS & $20(71.4 \%)$ & $16(57 \%)$ \\
Non-specific dysfunctions & $9(100 \%)$ & $2(22.2 \%)$ \\
Burst suppression & $2(100 \%)$ & $2(100 \%)$
\end{tabular}

(IEDs: inter ictal epileptiform discharges, NCS: non-convulsive seizures)

\section{Discussion}

Few studies showed that limited channel recording with central or parietal electrodes identifies the majority of seizures. ${ }^{15}$ The neonatal EEG is spatially rich with distinct electrical activity recorded at electrodes placed only few centimeters apart. ${ }^{16}$ Different types of EEG analysis are available like amplitude integrated EEG, combined spectrum analysis and nonlinear methods. Neonatal seizure monitoring using nonlinear EEG analysis showed a specificity of $89.8 \%$ and sensitivity of $65.9 \%$ for the detection of seizure activity. ${ }^{17}$ Diveyan et al stated that limited channel bed side EEG combining amplitude integrated EEG could detect majority of electrical series in newborn infants. ${ }^{18}$ The reduction in seizure burden with the number of recording electrodes was, however relatively small, and was no different between 4 and 10 electrode montages. This suggests that while some seizures may be focal, the majority of seizures spread wider on the cortical surface. ${ }^{19}$ The best available benchmark is visual EEG interpretation by the human expert. ${ }^{20,21} \mathrm{We}$ analyzed the whole data systematically in different montages.

EEG pattern and symmetry are totally different in infants when compared to the adult recording. Only few comparison studies are available regarding the electrode placement system. So the question is very relevant that; whether we required to apply whole 10-20 electrode placement system in infant group. Identifying epileptiform abnormalities and treating non-convulsive seizures play

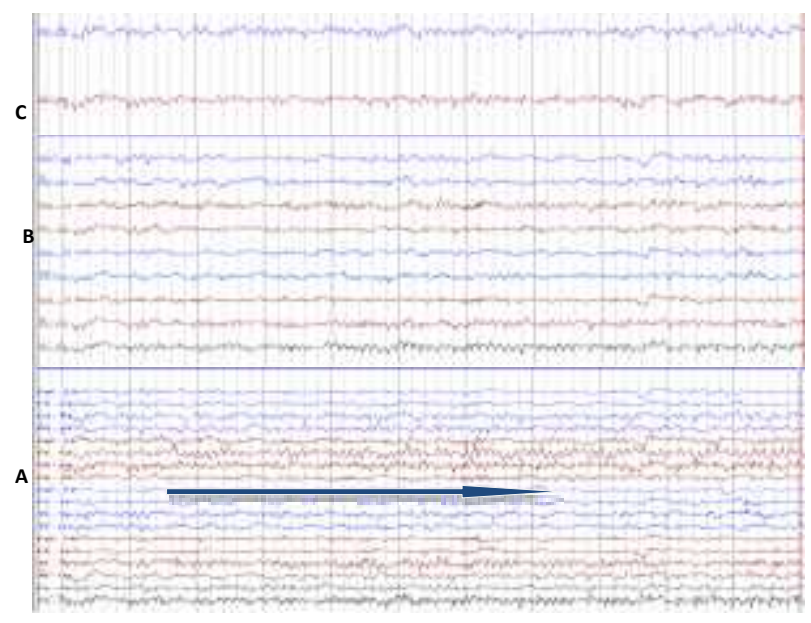

Figure 1.Seizures are seen in A, not in B and C 


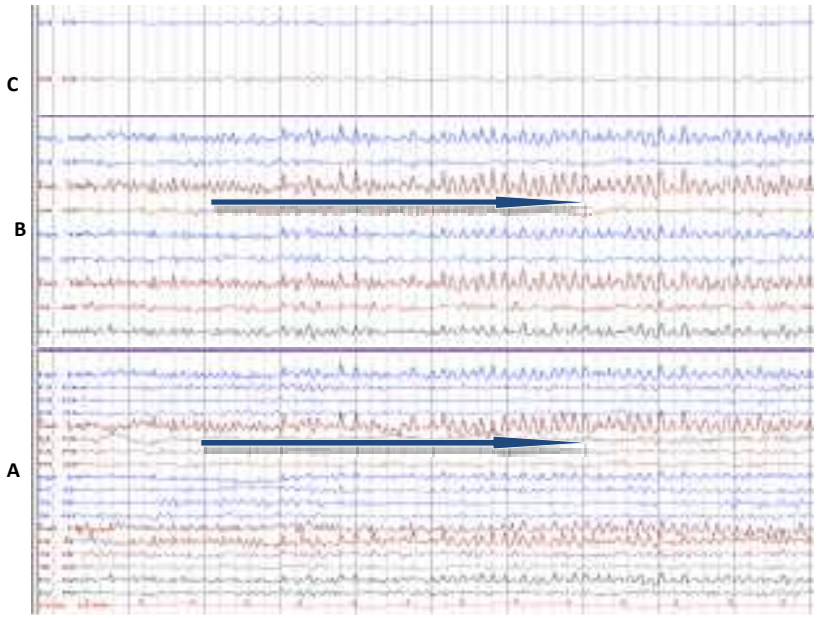

Figure 2. Seizure are seen in A and B; not in C

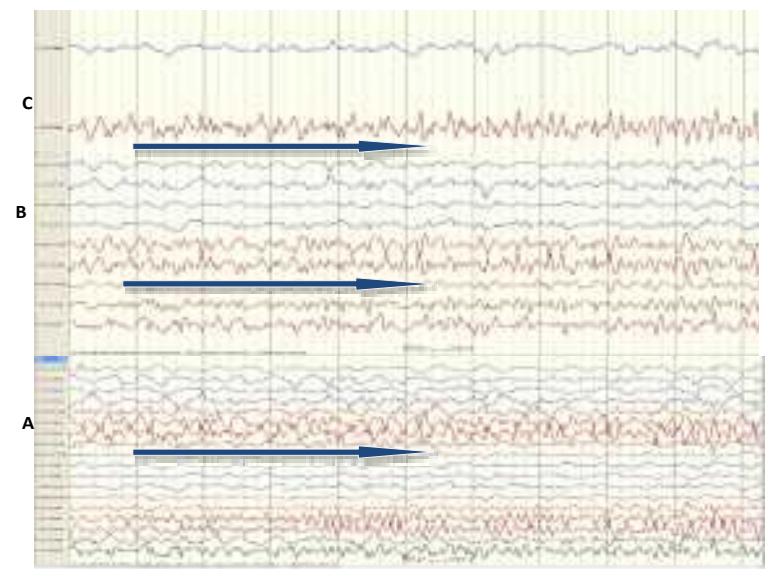

Figure 3. Seizures are seen in A, B and C

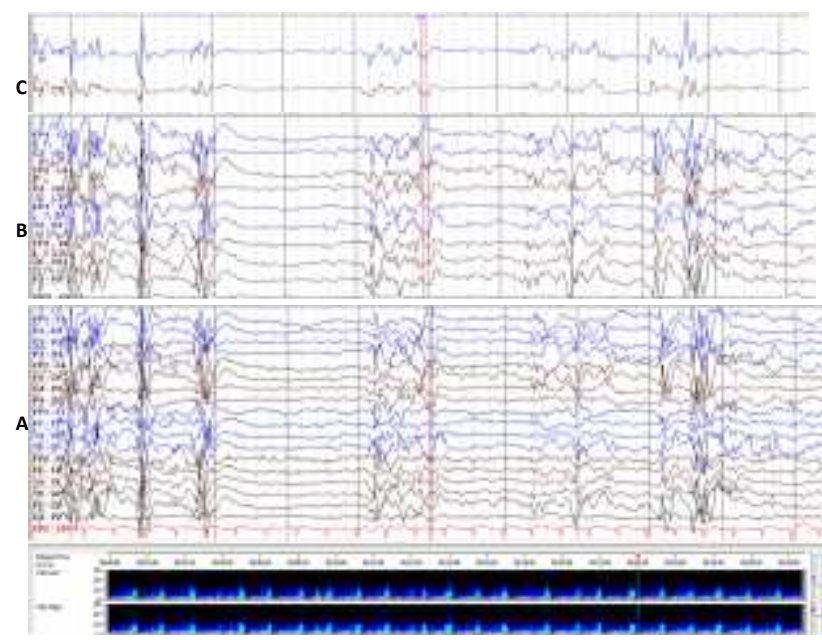

Figure 4. Burst-suppression pattern

duration seizures could be missed. But burst-suppression pattern, continuous and discontinuous patterns could easily identify in all three electrode placement systems.

We have done only routine 40 minutes EEG recording and we did not include continuous EEG monitoring data. Limited EEG monitoring may be helpful in the continuous EEG monitoring setting; where there are reduced technical resources in the form of man power but further studies may be done to ratify this end.

\section{Conclusion}

Standard EEG electrode placement has higher yield in detecting epileptiform abnormalities in routine EEG recording in pediatric population. Many of the epileptiform abnormalities could be missed in modified version of electrode placement.

\section{Acknowledgement}

The researchers would like to thank all Neurotechnologists of Ananthapuri hospitals and research institute.

\section{References}

1. N.S. Abend, A. Gutierrez-Colina, H. Zhao, R. Guo, E. Marsh, R.R. Clancy, et al. Inter observer reproducibility of electroencephalogram interpretation in critically ill children. J Clin Neurophysiol; 2011. 28:15-19. PMID: 21221016. PMCID: PMC3107383. DOI: $10.1097 /$ WNP.0b013e3182051123

2. N.S. Abend, A.A. Topjian, A.M. Gutierrez-Colina, M. Donnelly, R.R. Clancy, D.J. Dlugos Impact of continuous EEG monitoring on clinical management in critically ill children. Neurocritical Care; 2011.15:70-75. PMID: 20499208. PMCID: PMC3134111. DOI: 10.1007/s12028-010-9380-Z

3. S.L. Bonifacio, H.C. Glass, S. Peloquin, D.M. Ferriero A new neurological focus in neonatal intensive care. Nat Rev Neurol; 2011. 7:485-494. PMID: 21808297. DOI: 10.1038/nrneurol.2011.119

4. H.C. Glass, D.H. Rowitch The role of the neurointensive care nursery for neonatal encephalopathy. Clin Perinatol; 2016. 43:547-557. PMID: 27524453. PMCID: PMC4988330.

DOI: $10.1016 /$ j.clp.2016.04.011

5. P. Srinivasakumar, J. Zempel, S. Trivedi, M. Wallendorf, R. Rao, B. Smith, et al. Treating EEG seizures in hypoxic ischemic encephalopathy: A randomized controlled trial. Pediatrics; 2015.136:e1302-e1309. PMID: 26482675.

DOI: $10.1542 /$ peds.2014-3777

6. L.G. van Rooij, L.S. de Vries, A.C. van Huffelen, M.C. Toet Additional value of two-channel amplitude integrated EEG recording in full term infants with unilateral brain injury. Arch Dis Child-Fetal Neonatal Ed; 2010. 95:F160-F168. PMID: 19815938.

DOI: $10.1136 /$ adc.2008.156711

7. R.A. Hrachovy, E.M. Mizrahi Atlas of neonatal electroencephalography (4th ed.). Demos Medical, New York; 2015.

8. R.A. Shellhaas, T. Chang, T. Tsuchida, M.S. Scher, J.J. Riviello, N.S. Abend, et al. The American Clinical Neurophysiology Society's guideline on continuous electroencephalography monitoring in neonates. J Clin Neurophysiol; 2011. 28:611-617. PMID: 22146359. DOI: 10.1097/WNP.0b013e31823e96d7

9. R.A. Shellhaas, R.R. Clancy Characterization of neonatal seizures by conventional EEG and 
singlechannel EEG. Clin Neurophysiol; 2007. 118:2156-2161 PMID: 17765607

DOI: 10.1016/j.clinph.2007.06.061

10. C.J. Wusthoff, R.A. Shellhaas, R.R. Clancy Limitations of single-channel EEG on the forehead for neonatal seizure detection. J Perinatol; 2009. 29:237242. DOI: $10.1038 /$ jp.2008.195

11. G.B. Boylan, N.J. Stevenson, S. Vanhatalo Monitoring neonatal seizures. Fetal Neonatal Med; 2013. 18:202-208. PMID: 23707519. DOI: 10.1016/j.siny.2013.04.004

12. S. Vanhatalo, M. Metsäranta, S. Andersson Highfidelity recording of brain activity in the extremely preterm babies:feasibility study in the incubator. Clin Neurophysiol; 2008. 119:439-445.

DOI: https://doi.org/10.1016/j.clinph.2007.10.008

13. M Seeck M, L Koessler, T Bast et al The standardized EEG electrode array of the IFCN. Clinical neurophysiology; 2017. 128(10):2070-2077. PMID: 28778476. DOI: 10.1016/j.clinph.2017.06.254

14. R.R. Clancy, A. Legido, D. Lewis Occult neonatal seizures Epilepsia; 1988. 29:256-261. PMID: 3371282 DOI: 10.1111/j.1528-1157.1988.tb03715.X

15. M.D. Bourez-Swart, L. van Rooij, C. Rizzo, L.S. de Vries, M.C. Toet, T.A. Gebbink, et al. Detection of subclinical electroencephalographic seizure patterns with multichannel amplitude-integrated EEG in fullterm neonates. Clin Neurophysiol; 2009. 120:19161922. PMID: 19782640

DOI: 10.1016/j.clinph.2009.08.015
16. M. Odabaee, W.J. Freeman, P.B. Colditz, C. Ramon, S. Vanhatalo Spatial patterning of the neonatal EEG suggests a need for a high number of electrodes. Neuroimage; 2013. 68:229-235. PMID: 23246993. DOI: $10.1016 /$ j.neuroimage.2012.11.062

17. L.S. Smit, R.J. Vermeulen, W.P. Fetter, R.L. Strijers, C.J. Stam Neonatal seizure monitoring using nonlinear EEG analysis. Neuropediatrics; 2004.35:329335. PMID: 15627940. DOI: 10.1055/s-2004-830367

18. D.K. Shah, M.T. Mackay, S. Lavery, S. Watson, A.S. Harvey, J. Zempel, et al.Accuracy of bedside electroencephalographic monitoring in comparison with simultaneous continuous conventional electroencephalography for seizure detection in term infants. Pediatrics; 2008. 121;1146-1154. DOI: https://doi.org/10.1542/peds.2007-1839

19. N.J. Stevenson, R.R. Clancy, S. Vanhatalo, I. Rosén, J.M. Rennie, G.B. Boylan Interobserver agreement for neonatal seizure detection using multichannel EEG. Ann Clin Translat Neurol; 2015.2:1002-1011. DOI: https://doi.org/10.1002/acn3.249

20. M.S. Scher, Q.H. Bao, S. Mazumdar, M.J. Painter, J. Alvin, C.K. Redmond A reliability study of a neonatal seizure scoring system. J Epilepsy; 1994. 7:273-278. DOI: 10.1016/0896-6974(94)90056-6

21. C.J. Wusthoff, J. Sullivan, H.C. Glass, R.A. Shellhaas, N.S. Abend, T. Chang, et al. Interrater agreement in the interpretation of neonatal electroencephalography in hypoxic-ischemic encephalopathy. Epilepsia; 2017. 58:429-435. PMID: 28166364. PMCID: PMC5339031 DOI: $10.1111 /$ epi.13661 